Reprod. Nutr. Dévelop., 1988, 28 Suppl. n¹, 121-122

\title{
Influence du floconnage du blé sur le flux intestinal d'azote chez la vache
}

M. FOCANT, B. ALLART, Annick VAN HOECKE, M. VANBELLE

Université Catholique de Louvain, Faculté des Sciences Agronomiques, Unité de Biochimie de la Nutrition.

3, Place Croix du Sud, B-1348 Louvain-la-Neuve, Belgique.

Summary. Six Friesian heifers fed a $75 \%$ wheat diet were used in a $6 \times 2 \times 2$ cross-over design. Rolled and steam-flaked wheat were compared. Starch gelatinization failed to increase the DM intestinal flow. Decreased $\mathrm{N}$ solubility produced by steam-flaking was associated with an increase in Non-NH3-N flow $( \pm 12 \% ; \mathrm{P}<0.05)$.

Le traitement hydrothermique de l'orge, en assurant, dans le rumen, une protection des protéines contre la désamination, permet d'accroître, chez le mouton, les quantités d'azote transitant en 24 heures dans l'abomasum (Durand et al., 1974). Nous avons vérifié, chez le bovin, l'influence du floconnage du blé sur le flux d'azote duodénal.

Matériel et méthodes. Six génisses Frisonnes, d'un poids moyen de $491 \mathrm{~kg}$, munies d'une canule du rumen et d'une canule simple duodénale, ont été réparties en 2 lots selon un schéma expérimental en "cross-over ». Elles ont reçu, chaque jour, $1 \mathrm{~kg}$ de paille de blé hachée, $0,2 \mathrm{~kg}$ d'un complément minéral vitaminé, $0,075 \mathrm{~kg}$ d'urée et $4 \mathrm{~kg}$ de blé aplati ou floconné, en 2 repas distribués à $9 \mathrm{~h}$ et à $16 \mathrm{~h}$. Le floconnage, réalisé dans une installation "Buhler», a consisté en une cuisson à la vapeur à pression atmosphérique d'une durée de $45 \mathrm{~min}$, suivie d'un aplatissage à chaud. Des mélanges d'amidon de blé natif et d'amidon de blé complètement gélatinisé ont servi à établir une droite d'étalonnage mettant en relation le \% d'amidon gélatinisé et la quantité de sucres libérés par la pancréatine porcine après $30 \mathrm{~min}$ de digestion. Cette relation a permis d'estimer le degré de gélatinisation de l'amidon des échantillons testés. La dégradation in vitro des matières azotées a été déterminée après une incubation de 6 heures à $40^{\circ} \mathrm{C}$ dans un mélange tampon phosphate/liquide de rumen de mouton dans le rapport 4/1. Le flux du chyme duodénal a été établi par double marquage ( $P E G$ et $\mathrm{Cr}_{2} \mathrm{O}_{3}$ ) (Faichney, 1975) sur tous les animaux et sur deux jours.

Résultats et discussion. Le traitement hydrothermique du blé a conduit à la gélatinisation complète de $80 \%$ de l'amidon et à la diminution de la dégradation in vitro des matières azotées ( 9 au lieu de $62 \%$ ). Cependant, cette insolubilisation des protéines dans le liquide de rumen n'est pas associée à une moindre digestion par la pepsine (tabl. 1).

Le traitement de l'amidon ne modifie pas le flux de matière sèche au niveau du duodénum (tabl. 2). Ceci est logique puisque pour des régimes contenant 
TABL. 1. - Caractéristiques des blés distribués.

\begin{tabular}{lcc}
\hline & Blé aplati & Blé floconné \\
\hline Amidon (\% MS) & 67,4 & 68,4 \\
Amidon gélatinisé (\% amidon) & 0,0 & 80,1 \\
$\mathrm{~N} \times 6,25(\% \mathrm{MS})$ & 14,4 & 14,2 \\
$\mathrm{~N}$ dégradé par la pepsine $(\% \mathrm{~N})$ & 95,7 & 93,3 \\
$\mathrm{~N}$ dégradé in vitro $(\% \mathrm{~N})$ & 61,9 & 8,8 \\
\hline
\end{tabular}

entre 70 et $80 \%$ de blé aplati, la digestibilité de l'amidon dans le rumen des bovins est de l'ordre de $95 \%$ (Thivend et Journet, 1968) ; une telle digestion est difficile à améliorer. Par contre, le flux d'azote non ammoniacal est augmenté de $12 \%$ par le floconnage $(P<0,05)$. Ce résultat confirme pour le blé, ceux observés pour l'orge, par Durand et al. (1974) et par Papasolomontos et Wilkinson (1976) chez les ovins, et par MacMeniman (1975) chez les bovins.

TABL. 2. - Influence du floconnage du blé sur le flux intestinal de matière sèche et d'azote non ammoniacal chez la vache $(n=6)$.

\begin{tabular}{lccc}
\hline Régime & Blé aplati & Blé floconné & Stat. \\
\hline Matière sèche ingérée $(\mathrm{g} / \mathrm{j})$ & 4695 & 4707 & - \\
$\mathrm{N}$ ingéré $(\mathrm{g} / \mathrm{j})$ & 121 & 120 & - \\
Flux de matière sèche $(\mathrm{g} / \mathrm{j})$ & $2792 \pm 188$ & $2823 \pm 141$ & $\mathrm{~N} . \mathrm{S}$. \\
Flux d'N non NH3 $(\mathrm{g} / \mathrm{j})$ & $98 \pm 6$ & $110 \pm 8$ & $\mathrm{P}<0,05$ \\
\hline
\end{tabular}

En conclusion, le floconnage du blé après cuisson à la vapeur permet d'accroître, chez la vache, les quantités d'acides aminés entrant dans l'intestin grêle. Cette amélioration peut résulter de la plus faible dégradation des protéines alimentaires et/ou d'une synthèse microbienne accrue dans le rumen.

Remerciements. - Travail subventionné par I'IRSIA, rue de Crayer, 6, B-1050 Bruxelles, et par la Soc. Coop. INTERAGRI, Parc Industriel, B-5210 Seilles.

Durand M., Ben Ameur M., Viroben G., 1974. Ann. Biol. anim. Bioch. Biophys., 14, 167-192.

Faichney G. J., 1975. 277-291. In McDonald I. W., Warner A. C. I., Proc. I/th int. Symp. Rumin. Physiol., Univ. New England, Armidale, Australia.

MacMeniman N. P., 1975. PhD Thesis, University of Newcastle-upon-Tyne.

Papasolomontos S. A., Wilkinson J. I. D., 1976. 31-60. In Optimising the utilisation of cereal energy by cattle and pigs, Tech. Pub. U.S. Feed Grains Council, London.

Thivend P., Journet M., 1968. Ann. Biol. anim. Bioch. Biophys., 8, 449-455. 